\title{
Diseño y evaluación de un juego serio para la formación de estudiantes universitarios en habilidades de trabajo en equipo
}

\author{
Raquel Poy-Castro ${ }^{1}$, Cristina Mendaña-Cuervo ${ }^{1}$, Begoña González ${ }^{1}$ \\ rpoyc@unileon.es, cristina.mendana@unileon.es, bgonzalez@unileon.es \\ ${ }^{1}$ Universidad de León, Av. de la Facultad de Veterinaria, 25, 24004 León, Spain
}

DOI: 10.17013/risti.e3.71-83

\begin{abstract}
Resumen: Este trabajo se centra en el análisis de la adquisición y el desarrollo de ciertas competencias genéricas en un grupo de estudiantes de Grado mediante el diseño de una nueva actividad de aprendizaje, específicamente un Juego Serio. A partir de la exigencia de nuevos métodos de enseñanza y, sobre todo aquellos relacionados con las tecnologías de aprendizaje, en el Espacio Europeo de Educación Superior (EEES), el uso de juegos serios se justifica porque promueven el aprendizaje y transferencia de conocimiento. En concreto, se ha diseñado un juego serio, Concordia, con el fin de promover y analizar el desarrollo de la habilidad de trabajo en equipo. En este juego serio, los estudiantes tienen que resolver un problema cuya solución depende principalmente de la capacidad de negociación entre los miembros del grupo. Los resultados muestran que la experiencia mejora la solución final del juego y la habilidad de trabajo en equipo.
\end{abstract}

Palabras clave: Software educativo; estudiantes de Grado; formación; habilidades de trabajo en equipo; juego serio.

\section{Designing and evaluating a serious game for training university students in team-working skills}

Abstract: This paper is focused on analyzing the acquisition and development of certain generic skills in a group of degree students by means of the design of a new learning activity, specifically a Serious Game. New teaching methods and above all those ones related to learning technologies are demanded in European Higher Education Area (EHEA). The use of Serious Games is justified because they promote learning y knowledge transfer. Specifically we have designed a Serious Game, Concordia, in order to promote and analyze the development of the teamwork skill. In this Serious Game, students have to solve a problem whose solution mainly depends on the ability to negotiate among group members. Thus, we wanted to know if students can improve their teamwork skill with this Serious Game. The results show that the experience improves the final solution of the game, so this Serious Game improves the teamwork skill.

Keywords: Educational software; Degree students; training; teamwork skills; serious game. 


\section{Introducción ${ }^{1}$}

El aprendizaje basado en juegos serios en el ámbito educativo es una incorporación reciente, producto de la proliferación de ayudas tecnológicas en el ámbito educativo y de la apertura necesaria a nuevos métodos didácticos que apoyen al educador en la complejidad de aunar la transmisión de conocimientos y en el entrenamiento en competencias que comprenden el proceso de aprendizaje.

En los últimos años se ha producido un notable conjunto de experiencias de aplicación del aprendizaje basado en juegos a escenarios de formación reglada, particularmente en el entorno de la educación superior, que han animado a intentar desarrollar una herramienta de trabajo diseñada para el desarrollo de una de las competencias más complejas como objetivo del proceso de aprendizaje y más reñida a la vez con el individualismo: el trabajo en equipo (Westera, Nadolski, Hummel \& Wopereis, 2008; Guillen-Nieto y Aleson-Carbonell, 2012).

\subsection{Establecimiento de la importancia del tema}

El proyecto que se ha desarrollado se circunscribe dentro de la regulación española del sistema de educación superior, partiendo del Real Decreto 1393/2007 por el que se establece la ordenación de las enseñanzas universitarias oficiales, cuya finalidad debe ser la obtención por parte del estudiante de una formación general orientada para el ejercicio de actividades de carácter profesional. Esta orientación profesional requiere integrar en las enseñanzas competencias genéricas básicas y competencias más específicas que posibiliten una orientación profesional que permita a los titulados la integración en el mercado de trabajo.

Ello obedecería a que tradicionalmente los graduados eran personas que habían completado sus estudios, demostrando sus progresos en un ámbito de conocimiento específico, pero en la actualidad los estudiantes tienen además que demostrar la adquisición de determinadas competencias que tendrán que aplicar en la práctica de actividades profesionales o de investigación relacionadas con su ámbito de estudio (González y Wagenaar, 2003; Rue y Martínez, 2005; Delgado García, 2005).

En un marco más amplio, la evolución actual de la Sociedad del Conocimiento exige un cambio de mentalidad en el profesorado para adaptar las metodologías docentes, en base a las posibilidades que ofrece el entorno digital para enseñar de forma distinta, adaptándose a las formas de aprender de los "nativos digitales". Enseñar de forma diferente exige el empleo de metodologías activas y cooperativas para un mundo lleno de complejidad. Y precisamente para trabajar de forma cooperativa, es necesario desarrollar la competencia de trabajo en equipo, que existe cuando los miembros del grupo perciben que están unidos entre sí (interdependencia positiva) de forma que no pueden triunfar sin que los demás triunfen (responsabilidad individual y grupal), debiendo de existir una interacción que tiene lugar cuando los miembros del grupo comparten recursos, ayuda, apoyo y aprenden habilidades sociales que les permitan actuar como parte del grupo. Para

\footnotetext{
${ }^{1}$ Esta investigación ha sido soportada económicamente por la Universidad de León a través de su Convocatoria de ayudas a Proyectos de Innovación Docente desarrollándose entre diciembre de 2012 y diciembre de 2013.
} 
ello, los grupos deben hablar sobre el proceso, y sobre cómo van a conseguir los objetivos. Por último, deben detectar qué acciones de los miembros del grupo ayudan o no y decidir qué conductas cambiar y cuáles no, lo que se conoce como autoevaluación grupal.

Siguiendo a Domingo (2008) por aprendizaje cooperativo se entiende "el poder operar, desde un punto de vista docente, con pequeños grupos de estudiantes que trabajan juntos para aprovechar al máximo su aprendizaje y el de sus compañeros de grupo, de manera que cada cual pueda construir su conocimiento si dispone de colaboración de sus compañeros". Es precisamente en este ámbito en el que se circunscriben los denominados juegos serios, los cuales promueven la comprensión, la integración y la aplicación de conceptos, lo que permite mejorar el rendimiento, a diferencia de las clases magistrales en las que el alumno es un mero receptor de la información (Clark, 2004; Pease, 2010). Desde esta perspectiva, la participación de los estudiantes en entornos virtuales, donde tienen que poner en práctica su aprendizaje, implica un refuerzo de la experiencia de enseñanza-aprendizaje (Squire, 2005; Sharpe, Beetham y De Freitas, 2010).

Lo anteriormente expuesto cobra pleno sentido si tenemos en cuenta el nuevo escenario de la Universidad de León, que apuesta por el estímulo en los docentes de mejorar la calidad de la docencia acorde con la nueva coordenada contextual de la acción docente enmarcada en el Espacio Europeo de Educación Superior (EEES), en la que se hace necesario el desarrollo de programas de innovación docente que estimulen y reconozcan la participación del profesorado en actividades que desarrollen nuevas metodologías de enseñanza-aprendizaje, que en este caso se centrarían en propiciar el desarrollo de competencias genéricas en los alumnos a través del diseño de actividades de aprendizaje basadas en Juegos Serios (Serious Games).

La utilización de los Juegos Serios está justificada por la capacidad que ofrecen de simular la realidad, lo que los convierten en una herramienta clave para promover el aprendizaje y transferir el conocimiento, estimulando la participación de los estudiantes en escenarios virtuales, lo que sin ninguna duda fomenta la generación y manejo de expectativas, comenzando por la voluntad de los participantes, por aprender.

\subsection{Objetivos específicos del proyecto e hipótesis}

Para poder entender el objetivo específico, se recurrió a una metáfora. En el filme de John Badham (1983) "Juegos de Guerra", David (Mathew Broderick) es un experto informático capaz de saltarse los más avanzados sistemas de seguridad y de descifrar los más herméticos códigos secretos, si bien su juego se complica cuando involuntariamente conecta su ordenador al Departamento de Defensa americano, encargado del sistema de defensa nuclear. Desencadena así una situación de peligro difícilmente controlable. Con la ayuda de su novia y de otro informático genial intentará, en una carrera contrarreloj, evitar la Tercera Guerra Mundial. Esta idea sugirió diseñar un juego serio que permitiera poner a estudiantes en una situación de estrés que provocase la necesidad de analizar en grupo y detectar una estrategia que involucrase al completo las habilidades de trabajo en equipo para su resolución, fruto de la cual surgió Concordia.

Como hipótesis de investigación se sostiene que la mejora de trabajo en equipo mejora con la experiencia en dicho juego serio. Para ello se ha tomado en consideración que los escenarios de aprendizaje mediados por las TIC, con base en tecnologías digitales 
interactivas, puede favorecer las condiciones para la puesta en práctica de las nuevas metodologías activas, reclamadas desde todos los foros educativos: se hacen viables diversas modalidades de aprendizaje abierto, con ofertas educativas flexibles, válidas tanto para entornos presenciales como semipresenciales o a distancia (Carrillo Ramos, 2009; Cook, Ley, Crawford y Warner, 2009).

\section{Metodología}

Para entender las razones que justifican la elección del método se hace uso de una segunda metáfora. En la película "Airplane" (Aterriza como puedas), producida y dirigida por Jim Abrahams, David Zucker y Jerry Zucker (1980), Ted Striker, un ex piloto de caza, debe vencer su miedo a volar, causado por un trauma psicológico sufrido durante la guerra, y tomar los mandos del avión, conducirlo a su destino y hacerlo aterrizar en malas condiciones atmosféricas, guiado por los controladores de tráfico aéreo Steve McCrosky (Lloyd Bridges) y Rex Kramer (Robert Stack) y con la ayuda de su ex novia Elanie (Julie Hagerty), azafata a bordo, y la de uno de los pasajeros, el estrafalario médico Dr. Rumack (Leslie Nielsen). Esta situación de tensión provocada por la necesidad de aterrizar era precisamente la emulación que deseábamos provocar con nuestro juego serio Concordia.

Se trataba de una herramienta atípica y sorprendente para alumnos que no esperaban en ningún caso afrontar el reto de resolver una situación que podría ser real: convertirse en equipos de controladores aéreos. En consecuencia, se abordo el diseño del juego desde el punto de vista de una herramienta de simulación adaptada a un entorno de nativos digitales no expertos pero familiarizados con los juegos digitales. Para ello, se contó con la dirección técnica de Jesús Gonzalo (coordinador de la Titulación en Ingeniería Aeronáutica de la ULE) asistido directamente por un equipo de expertos en diseño Pedagógico.

Las carencias en el diseño pedagógico constituyen uno de los nuevos y principales retos del uso de juegos serios en entornos educativos. De este modo, se aplicó el diseño propuesto y posteriormente la evaluación al Marco de Análisis desarrollado por el Grupo de Investigación Aplicada del Instituto de Juegos Serios de la Universidad de Coventry (De Freitas y Jarvis, 2008; De Freitas et al., 2009; Jarvis y De Freitas, 2009). Esta herramienta resulta de utilidad para los profesores que usan los juegos y simulaciones en el aprendizaje y enseñanza práctica, e incluye las cuatro dimensiones de análisis siguientes: a) los aspectos específicos del aprendiz: incluyendo el perfil, rol y competencias, b) la pedagogía utilizada: asociativa, cognitiva y social-situacional, c) la representación seleccionada para el diseño del juego: fidelidad, interactividad, grado de inmersión, y d) el contexto en el que se desarrolla el aprendizaje: ambiente, acceso al aprendizaje y los recursos de apoyo.

Por otro lado, al igual que el juego siempre ha supuesto una actividad inherente a la vida escolar, especialmente en el ámbito de la educación no formal o informal, sin embargo, no se ha abordado el necesario paso a la integración del juego en la educación formal, siendo relegado a menudo como actividad distractora del propósito 
educativo, desaprovechando su potencial instructivo. Gee (2005) señala que, pese a que el aprendizaje se basa en la motivación de los estudiantes por parte de sus docentes (empowerment) y una combinación entre la capacidad de resolver problemas y de conseguir conocimiento sobre la realidad, sin embargo los participantes en un juego juegan para satisfacer una necesidad social y no tanto para resolver el desafío de un juego. De este modo, a menudo se obvia que la práctica de juegos digitales en el aula no sólo consiste en la puesta en práctica de un aprendizaje de conocimientos, sino que supone la aplicación de comportamientos en orden a una estimulación del más amplio conjunto cognitivo del triángulo conocimientos-actitudes-percepciones.

Por último, se pretendido contribuir a una aproximación académica al ámbito del diseño pedagógico de los juegos serios en el aula, dado que en el ámbito académico la mayoría de los estudios hasta la fecha se han centrado especialmente en el estudio de la interacción tecnológica y, en menor medida, en los aspectos pedagógicos y de soporte a los estudiantes (Pivec, 2007).

\subsection{Descripción del método utilizado para el diseño del software}

En relación al diseño del juego serio cabe mencionar que se abordó un diseño instruccional en orden a los requisitos organizacionales y de usuario, teniendo en consideración los siguientes parámetros:

- Debía tratarse de una herramienta de fácil manejo y comprensión para estudiantes de cualquier tipo de formación o conocimientos previos.

- Debía proponer tareas que requiriesen ejercicios colaborativos entre varios jugadores.

- En cuanto al diseño del software, se optó por las características siguientes:

No debía tratarse de un juego competitivo estilo "arcade".

- Se debían evitar elementos confusos en el ámbito del lenguaje utilizado, los gráficos o el diseño, por lo que se optó por un estilo bidimensional.

- Se evitaron elementos alusivos a valores sociales o éticos que provocasen reacciones imprevistas en el plano emocional de los jugadores.

- Se escogió un diseño con simplicidad de perspectivas.

En consecuencia, se optó por recurrir a un diseño basado en experiencias de simulación (Simulation Experience Design Method) que se han demostrado como instrumentos de entrenamiento con un elevado potencial para la puesta en práctica de capacidades por parte del jugador, tanto por la combinación del factor "tiempo real” como por el retorno de resultados para el jugador (Raybourn 2006; Knight et al., 2010).

El resultado fue el diseño de Concordia, un juego serio que simula una actividad de control del tráfico aéreo, en el que los jugadores tienen que combinar una serie de aviones en vuelo, que tienen diferentes rutas y duración del vuelo, para optimizar el uso del espacio aéreo (medido en el tiempo) y evitar cualquier colisión entre ellos. Cada miembro del equipo representa una aerolínea y es responsable de 3 aviones, por lo que cada equipo debe colocar 12 aviones en el espacio aéreo. Tienen que encontrar la mejor solución posible para reducir al mínimo el tiempo de vuelo total de todos sus aviones. 
Los vuelos de cada línea aérea tienen el mismo color y todos ellos se pueden mover a lo largo de un eje que representa el tiempo de vuelo. Cada plano es un segmento, cuya pendiente y longitud representan, respectivamente, la ruta y la duración del vuelo. En la Figura 1 se representa el problema inicial.

Como resultado se pueden dar varias combinaciones de colocación de los aviones que permiten evitar las colisiones entre ellos. Por ejemplo, las Figuras 2 y 3 representan dos posibles soluciones para el problema inicial. Lógicamente, la mejor solución es la combinación de aviones que permite la más corta duración total del vuelo.

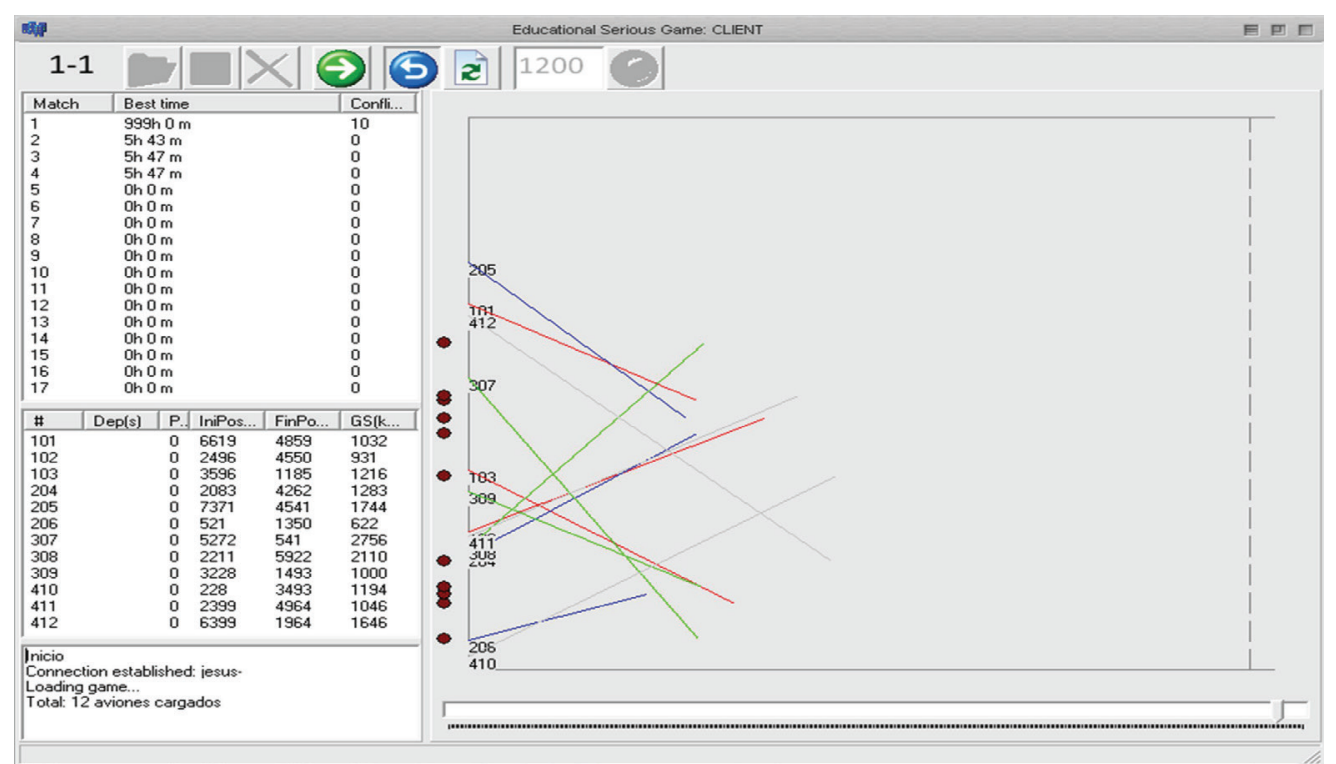

Figura 1 - Problema inicial planteado por el juego Concordia. 


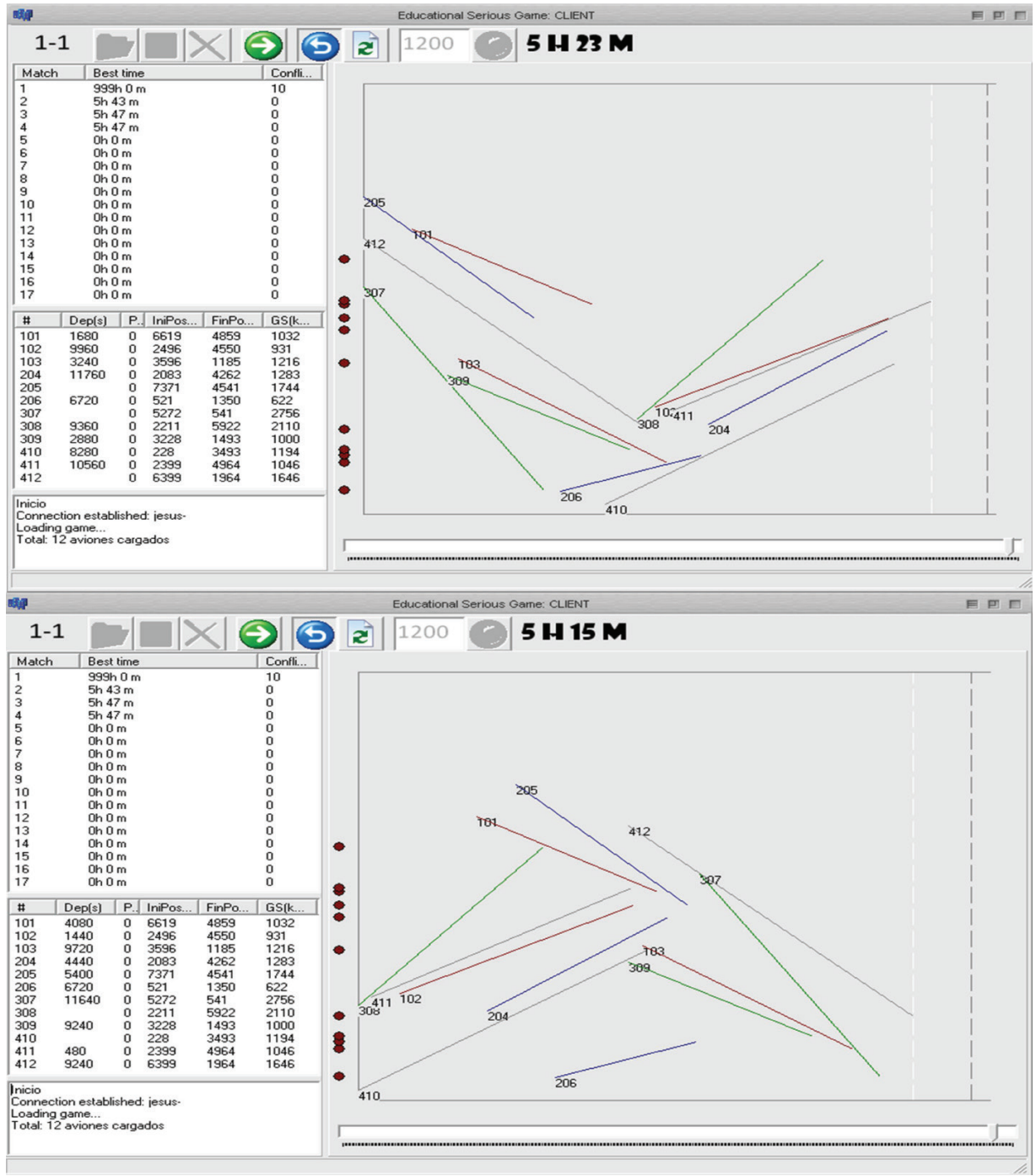

Figura 2 - Soluciones posibles ante el problema inicial.

De este modo, cada miembro del equipo tiene que negociar sobre la distribución de los aviones dentro del espacio aéreo con los demás miembros del equipo, ya que su mejor solución viene necesariamente apoyada por las soluciones de sus compañeros de grupo, en consonancia con la idea base de la competencia de trabajo en equipo. Dado que la mejor solución es conocida por el profesorado, es posible conocer el número de grupos que efectivamente a través del proceso de negociación consiguen obtenerla. 


\subsection{Descripción de las características de la muestra}

El proyecto se lleva a cabo durante el período Septiembre 2011 a enero de 2011, con estudiantes del Grado en Educación Infantil de la Universidad de León. La muestra inicial fue de 63 estudiantes, de los cuales el número de participantes válidos fue 56 debido a la falta de asistencia a las sesiones u otras circunstancias. De este modo, los participantes fueron asignados al azar a 14 grupos de cuatro personas, los cuales realizaron cuatro sesiones de juego de diez minutos. En la siguiente Tabla 1 se detalla el perfil de los participantes.

Tabla 1 - Perfil de la muestra.

\begin{tabular}{lll}
\hline Edad & Número & \% \\
\hline $0-18$ & 21 & $37.50 \%$ \\
\hline $19-22$ & 25 & $44.64 \%$ \\
\hline $22-26$ & 8 & $14.29 \%$ \\
\hline $27+$ & 2 & $3.57 \%$ \\
\hline Género & Número & $\%$ \\
\hline Hombres & 6 & $10.71 \%$ \\
\hline Mujeres & 50 & $89.29 \%$ \\
\hline Total & 56 & $100 \%$ \\
\hline
\end{tabular}

\subsection{Descripción del proceso de recogida de datos}

La recogida de datos se llevó a cabo en varias sesiones. En la primera sesión se presenta el tutorial del juego y el sujeto se enfrenta a la ejecución de las primeras partidas individuales, en la segunda sesión los sujetos se familiarizaron con el funcionamiento del juego serio por equipos, y se clarifican los sistemas de puntuación, como paso previo a la realización de las primeras partidas de equipo, pero de modo no competitivo entre los diferentes equipos. La tercera y cuarta sesión se destinan íntegramente a realizar partidas en equipos, los que permite obtener los datos de las soluciones obtenidas.

Dado que el objetivo principal era el desarrollo de la competencia de trabajo colaborativo, se suponía que los estudiantes podrían obtener los mejores resultados sólo si el equipo de jugadores recurrían a técnicas de comunicación, negociación y cooperación entre ellos. En orden a analizarlo, las soluciones obtenidas por los 14 grupos en la primera ronda de juego (sin entrenamiento) se compararon con los participantes en el último juego (después del entrenamiento). Además, se utilizó una prueba de Chi cuadrado para analizar las dos variables categóricas: la experiencia del juego serio (con o sin entrenamiento previo) y la obtención del mejor resultado del juego (mejor tiempo respecto a otros tiempos registrados) y de este modo, en consecuencia, la mejora en la competencia de trabajo en equipo. 


\section{Resultados}

Los datos de los 14 grupos se muestran en la tabla de contingencia siguiente (Tabla 2). En la parte superior (sin entrenamiento), sólo 2 grupos obtuvieron el mejor rendimiento. En el juego final, después del entrenamiento, 8 equipos consiguieron la solución.

Tabla 2 - Resultados por grupo.

\begin{tabular}{llll}
\hline & \multicolumn{3}{c}{ TRAINING } \\
\cline { 2 - 3 } & Without & With & Total \\
\hline Best Time & 2 & 8 & 10 \\
\hline Other Time & 12 & 6 & 18 \\
\hline TOTAL & 14 & 14 & 28 \\
\hline
\end{tabular}

El valor de Chi cuadrado fue 5,6o con 1 grado de libertad, por lo que es estadísticamente significativa para un nivel de significación de 0,05. Por tanto, se acepta la hipótesis nula de independencia entre la formación (la experiencia en el juego serio) y tiempo de solución, que mide la mejora de las habilidades de trabajo en equipo. Además, o\% de las celdas tenían una frecuencia esperada inferior a 5, por lo que los resultados de la prueba pueden considerarse fiables.

\section{Conclusiones}

Este estudio pretende mostrar que los estudiantes que han recibido formación mediante el método de los Juegos Serios están más dispuestos a colaborar y establecer acciones de trabajo en equipo, gracias a la motivación adicional que supone la resolución de un desafío como el juego. Se trata de un hallazgo alentador aunque es necesario profundizar en la investigación para determinar cuánto tiempo de uso de los Juegos Serios resulta necesario para alcanzar los objetivos de la asignatura en cuanto a adquisición de competencias se requiere. De este modo podríamos averiguar si la efectividad del Juego Serio es compatible con el número de horas que requiere su práctica a efectos de conseguir los objetivos de la asignatura por parte del alumno.

Otra de las conclusiones de la experiencia, tras consultar a los alumnos participantes, es que efectivamente la motivación tanto para participar en este tipo de actividades inusuales para la formación reglada habitual no sólo es muy elevada, sino que igualmente resulta mayoritaria la disposición para volver a participar en actividades similares en la línea sugerida por Clark (2004).

Del resultado de aplicar la metodología de análisis de De Freitas y Oliver (2006), concluimos que, en relación a los aspectos específicos del aprendiz destaca la competencia de trabajo en equipo. El trabajo en equipo en este trabajo se comprende como la capacidad de que los estudiantes colaboren activamente en las tareas de equipo, fomentando la confianza, la cordialidad y la orientación a la tarea conjunta. Sería necesario un análisis más profundo para averiguar las implicaciones de la actividad lúdica en la adquisición de otras competencias ya que, por ejemplo, otros elementos trabajados 
en esta actividad involucraron además de las actividades colaborativas, la aplicación del pensamiento estratégico e innovador. Además, la proximidad de la experiencia formativa a la evaluación del ejercicio puede provocar un efecto multiplicador sobre el nivel de rendimiento de los participantes que suponga un sesgo en nuestras conclusiones.

En cuanto a la pedagogía usada, se ha centrado en aquellas actividades eminentemente de carácter comunicativo (Conole, 2007), actividades que aluden al empleo del diálogo para resolver los conflictos surgidos durante la resolución de las tareas, de modo que la dimensión social se sustenta en el intercambio entre los alumnos. En relación a la tercera y cuarta dimensiones hay que destacar que la representación seleccionada de un control aéreo ha facilitado la inmersión en el juego de los jugadores, facilitando el factor de tiempo real la interactividad entre los miembros del grupo en un contexto de aprendizaje muy dinámico y de corta duración

Mientras que existe una importante tradición en el estudio de las aplicaciones de los Juegos Serios en entornos comerciales o de entrenamiento profesional, con el estudio presentado en este trabajo se contribuye a la aplicabilidad en entornos de formación reglada para el desarrollo de competencias genéricas de colaboración entre los estudiantes.

De modo más general, este estudio pretende contribuir a una mejor comprensión del potencial de los juegos de simulación en la motivación de experiencias afectivas en los usuarios. La competencia de trabajo en equipo es necesaria en muchos entornos de aprendizaje y un sistema basado en juegos proporciona la capacidad de aprender, practicar y evaluar la competencia en un modo que no resulta fácil mediante los métodos tradicionales.

Este estudio ha demostrado que los juegos serios pueden ser utilizados con el fin de trabajar las habilidades, especialmente la habilidad de trabajo en equipo. Concordia, el juego serio que hemos diseñado, es una herramienta útil para la adquisición y desarrollo de habilidades transversales, específicamente la habilidad de trabajo en equipo, que es una competencia transversal para los estudiantes de grado dentro del EEES.

\section{Anexo 1. Cuestionario}

Esta encuesta forma parte del trabajo de investigación del Plan de Apoyo a la Innovación Docente "Los Juegos Serios como herramienta para la innovación docente en la adquisición, seguimiento y evaluación de las competencias transversales”.

La cumplimentación de la encuesta supone la autorización para que los datos sean tratados anónimamente con fines de investigación de forma exclusiva. 


\section{Datos personales y autorización}

\section{1: Código de usuario o nick:}

Por favor, escribe un código que puedas recordar, ya que deberás utilizarlo en posteriores sesiones.
2: Sexo:
$\Gamma$ Hombre
Mujer

3: Edad:

\section{Percepción del problema}

4: De la explicación teórica, chas sido capaz de extraer la regla o reglas que permiten encontrar rápidamente la solución?

$\Gamma$ Sí (pasa a la pregunta siguiente) $\Gamma$ No (pasa a la pregunta número 6)

\section{5: ¿Cuál crees que es la/s regla/s?. Escríbela/s}

6: Señala cuál de las siguientes variables crees que son necesarias para obtener una buena solución:

\begin{tabular}{|c|c|c|c|}
\hline ento gue renrese & $\begin{array}{l}\mathrm{Si} \\
\mathrm{C}\end{array}$ & $\begin{array}{l}\text { No } \\
\text { C }\end{array}$ & $\begin{array}{l}\mathrm{NS} / \mathrm{N} \\
\mathrm{C}\end{array}$ \\
\hline & C & $r$ & $r$ \\
\hline La pendiente del segmento que representa al avión & & & \\
\hline La situación en el eje de ordenadas & $E$ & D & $\mathrm{C}$ \\
\hline La pendiente y la longitud del segmento que representa al avió & C & B & C \\
\hline La situación en el eje de abscisas & C & C & $\mathrm{C}$ \\
\hline La situación en el eje de ordenadas & $\mathrm{C}$ & C & $\mathrm{C}$ \\
\hline Ninguna de las anteriores & $\mathrm{E}$ & $\mathbb{C}$ & C \\
\hline
\end{tabular}

7: En el caso del juego en equipo, ¿qué habilidades consideras más relevantes para obtener una buena solución?

Iniciativa propia

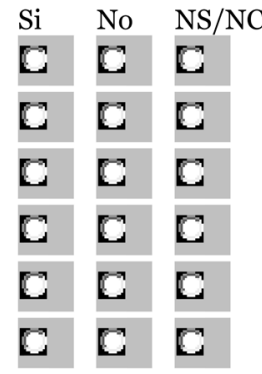

Cooperación con el resto de miembros del grupo

Comunicación asertiva positiva

Negociación entre los miembros del grupo

Competitividad con el resto de miembros del grupo

Comunicación asertiva negativa 


\section{Referencias}

Carrillo Ramos, A. (2009). Enseñanza de la metodología RUP de Ingeniería de Software. Biblioteca Virtual. Extraído desde http://www.eumed.net/libros/2009c/578/ index.htm

Clark, C.D. (2004). The Principles of Game Based Learning. Paper presented to NETC/ LSC Conference April 10-11, 2004 Crystal City, VA.

Conole, G. (2007) Describing learning activities: Tools y resources to guide practice. In H. Beetham y Sharpe (Eds.) Rethinking pedagogy for a digital age: Designing an delivering e-learning, pp.81-91, London:Routledge.

Cook, R. G., Ley, K., Crawford, C y Warner, A. (2009). Motivators y inhibitors for university faculty in disctance y e-learning. British Journal of Educational Technology, 40 (1), pp. 149-163.

De Freitas, S. y Oliver, M. (2006) How can exploratory learning with games y simulations within the curriculum be most effectively evaluated? Computers y Education, 46 (3), pp. 249-264.

De Freitas, S. y Jarvis, S. (2008). Towards a development approach for serious games. In T.M. Connolly, M. Stansfield, y E. Boyle (Eds) Games-based learning advancements for multi-sensory human-computer interfaces: Techniques y effective practices. IGI Global. Hershey, PA.

De Freitas, S., Rebolledo-Mendez, G., Liarokapis, F., Magoulas, G. y Poulovassilis, A. (2009) 'Developing an evaluation methodology for immersive learning experiences in a virtual world'. In Rebolledo-Mendez, G., Liarokapis, F.y de Freitas, S. (Eds) Proceedings of 2009 Conference in Games y Virtual Worlds for Serious Applications, IEEE pp 43-50.

Delgado García, A.B. (Coord). (2005). Competencias y diseño de la evaluación continua y final en el Espacio Europeo de Educación superior. Madrid: Dirección General de Universidades-MEC.

Domingo, J. (2008). El aprendizaje cooperativo. Cuadernos de Trabajo Social, 21, pp. 231-246.

Gee. J.P. (2005). "Learning by Design: good video games as learning machines". E-Learning, 2 (1), pp. 5-16.

González, J. y Wagenaar, R. (2003). Tuning Educational Structures in Europe. Final Report Phase One. Bilbao: Universidad de Deusto.

Guillen-Nieto, V. y Aleson-Carbonell, M. (2012). Serious games y learning effectiveness: The case of it's a deal! Computers y Education, 58, pp. 435-448.

Jarvis, S.y de Freitas, S. (2009). Evaluation of a Serious Game to support Triage Training: In-game Feedback y its effect on Learning Transfer. Proceedings of 2009 Conference in Games y Virtual Worlds for Serious Applications, IEEE. 
Knight, J.F.; Carley, S.; Tregunnac, B.; Jarvis, S., Smithies, J., de Freitas, S., Dunwelle, I. y Mackway-Jonesb, K. (2010). Serious gaming technology in major incident triage training: A pragmatic controlled trial. Resuscitation, 81, pp. 1175-1179.

Pease, M.A. (2010). Experimental investigation of the effectiveness of problem-based learning. New York: Columbia University.

Pivec, M. (2007). Play y learn: potentials of game-based learning. British Journal of Educational Technology, 38 (3), pp. 387-393.

Raybourn, E.M. (2006). Applying simulation experience design methods to creating serious game-based adaptive training systems. Interacting with computers, 19 (2), pp. 206-214.

Rue, J. y Martínez, M. (2005). Las titulaciones UAB en el Espacio Europeo de Educación Superior. Sistema Europeo de Transferencia de Créditos. Colección Eines, 1. Barcelona: Universidad Autónoma de Barcelona.

Sharpe, R., Beetham, H. y De Freitas, S. (2010). Rethinking learning for a digital age. How learners are shaping their own experiences. London: Routledge.

Squire, K. (2005). Game-Based Learning. An xLearn Perspective Paper. Masie Center eLearning Consortium. Extraído desde http://newmediaforlearning.com/research/ Game-Based_Learning.pdf.

Westera, W., Nadolski, R.J., Hummel, H.G.K. y Wopereis, I.G.J.H. (2008). Serious games for higher education: a framework for reducing design complexity. Journal of Computer Assisted Learning, 24, pp. 420-432. 